\title{
Salicylic acid attenuates virulence in endovascular infections by targeting global regulatory pathways in Staphylococcus aureus
}

\author{
Leon Iri Kupferwasser,, ${ }^{1,2}$ Michael R. Yeaman,,3 Cynthia C. Nast,, 3,4 \\ Deborah Kupferwasser, ${ }^{1}$ Yan-Qiong Xiong, ${ }^{1,3}$ Marco Palma, ${ }^{5}$ \\ Ambrose L. Cheung, ${ }^{5}$ and Arnold S. Bayer ${ }^{1,3}$
}

${ }^{1}$ Division of Infectious Diseases, St. John's Cardiovascular Research Center and the Research \& Education Institute,
Harbor-UCLA (University of California-Los Angeles) Medical Center, Torrance, California, USA
${ }^{2}$ St. Mary Medical Center, Long Beach, California, USA
${ }^{3}$ UCLA School of Medicine, Los Angeles, California, USA
${ }^{4}$ Department of Pathology, Cedars-Sinai Medical Center, Los Angeles, California, USA
${ }^{5}$ Department of Microbiology, Dartmouth Medical School, Hanover, New Hampshire, USA

Aspirin has been previously shown to reduce the in vivo virulence of Staphylococcus aureus in experimental endocarditis, through antiplatelet and antimicrobial mechanisms. In the present study, salicylic acid, the major in vivo metabolite of aspirin, mitigated two important virulence phenotypes in both clinical and laboratory $S$. aureus strains: $\alpha$-hemolysin secretion and fibronectin binding in vitro. In addition, salicylic acid reduced the expression of the $\alpha$-hemolysin gene promoter, bla, and the fibronectin gene promoter, $f n b A$. Transcriptional analysis, fluorometry, and flow cytometry revealed evidence of salicylic acid-mediated activation of the stress-response gene sigB. Expression of the sigBrepressible global regulon $\operatorname{sar} A$ and the global regulon $a g r$ were also mitigated by salicylic acid, corresponding to the reduced expression of the $b l a$ and $f n b A$ genes in vitro. Studies in experimental endocarditis confirmed the key roles of both sar $A$ and $\operatorname{sig} B$ in mediating the antistaphylococcal effects of salicylic acid in vivo. Therefore, aspirin has the potential to be an adjuvant therapeutic agent against endovascular infections that result from $S$. aureus, by downmodulating key staphylococcal global regulons and structural genes in vivo, thus abrogating relevant virulence phenotypes.

J. Clin. Invest. 112:222-233 (2003). doi:10.1172/JCI200316876.

\section{Introduction}

Staphylococcus aureus is a leading cause of serious systemic infections (e.g., endocarditis, pneumonia, abscesses, septicemia, and osteomyelitis) and often requires hospitalization and intensive antimicrobial therapy (1). The emergence of $S$. aureus resistance to multiple antibiotics, including semisynthetic penicillins, quinolones, and vancomycin, has hastened the need to design more effective antimicrobial regimens (2). Consequently, novel strategies for the therapy of such infections have been sought, including the targeting of known staphylococcal virulence factors for attenuation $(3,4)$. Delineating compounds that suppress structural or regulatory staphylococcal genes crucial for pathogenesis would potentially accomplish

Received for publication September 10, 2002, and accepted in revised form April 15, 2003.

Address correspondence to: Ambrose L. Cheung, Department of Microbiology, Dartmouth School of Medicine, Hanover, New Hampshire 03755, USA. Phone: (603) 650-1310; Fax: (603) 650-1362; E-mail: ambrose.cheung@dartmouth.edu. Marco Palma's present address is: Department of Microbiology, Cornell University Medical School, New York, New York, USA. Conflict of interest: The authors have declared that no conflict of interest exists. two goals: (a) enhancement of the efficacy of traditional antimicrobial therapy, and (b) reduction in the selective pressure that drives antibiotic resistance (5).

Salicylic acid, the major in vivo metabolite of aspirin, has been demonstrated to interfere with virulence factors at a transcriptional level in several viruses, including the respiratory syncytial virus and HIV-1. Furthermore, in mammalian and plant cells, this compound has been reported to impact intracellular signaling processes and the cytokine network (6, $7)$. We recently showed that treatment of an invasive experimental S. aureus infection (endocarditis) with intravenous aspirin caused a significant reduction in bacterial densities within target tissues (vegetations and kidneys), and that these effects are mediated by salicylic acid (8). We reasoned that salicylic acid may exert a direct effect on bacterial adhesins that are necessary for bacterial colonization and propagation in host tissues, or on staphylococcal global regulatory elements such as the staphylococcal accessory regulator, $\operatorname{sar} A$, and the accessory gene regulator, $a g r$. These two loci also coregulate genes that encode a variety of exoenzymes and exotoxins, including those responsible for cytolysis of host tissues (e.g., the $\alpha$-hemolysin gene, bla) $(9,10)$. 
In this study, we demonstrate that the observed antivirulence properties of aspirin in vivo are based on the direct antimicrobial effects of its metabolite, salicylic acid, and involve activation of the stress-induced transcription factor $\operatorname{sig} B$, leading to repression of the sarA and agr global regulons in S. aureus.

\section{Methods}

Bacterial strains, plasmids, and growth media. The bacterial strains and plasmids used in this study are listed in Table 1. These strains were selected to represent a broad range of methicillin-susceptible and methicillin-resistant phenotypes, as well as both clinical and laboratoryderived constructs. All the clinical strains were isolated from patients with serious human infections requiring hospitalization, such as wound infections and endocarditis. Erythromycin $(5 \mu \mathrm{g} / \mathrm{ml})$ and chloramphenicol $(10 \mu \mathrm{g} / \mathrm{ml})$ were used for selection of transformants and transductants in $S$. aureus, as indicated. Prior to in vitro experiments, $S$. aureus strains were grown at $37^{\circ} \mathrm{C}$ in plain tryptic soy broth (control cells) or in medium containing 30 or $50 \mu \mathrm{g} / \mathrm{ml}$ salicylic acid for 18 hours to the late postexponential phase on a rotary shaker.
These salicylic acid concentrations encompass readily achievable serum levels observed during therapy of experimental $S$. aureus endocarditis (11). No impact of salicylic acid on the 24-hour $S$. aureus growth kinetics was observed at these concentrations (data not shown). In addition, the $\mathrm{pH}$ of the bacteria-salicylic acid reaction mixtures did not change as a result of either final concentration of salicylic acid used.

Impact of salicylic acid on $S$. aureus adherence to fibrinogen and fibronectin. The fibrinogen- and fibronectin-binding capacities of $S$. aureus strains were evaluated by direct binding of washed bacterial cells $\left(5 \times 10^{3}\right.$ CFUs $)$ to solid-phase fibrinogen and fibronectin in six-well plates $(9,10)$. Plates were coated with $50 \mu \mathrm{g} / \mathrm{ml}$ fibrinogen or fibronectin (Sigma Chemical Co., St. Louis, Missouri, USA), incubated at room temperature for 24 hours, washed three times with PBS, blocked with blocking solution, and washed three more times. Bacterial cells $\left(5 \times 10^{3} \mathrm{CFUs}\right)$ were applied to individual wells and incubated for 1 hour at $37^{\circ} \mathrm{C}$ on a rotating platform. After removal of unbound cells by washing three times with PBS, $2 \mathrm{ml}$ of tryptic soy agar was overlain in all wells. After 24 hours of incubation at $37^{\circ} \mathrm{C}$,

Table 1

Bacterial strains and plasmids used in this study

S. aureus

RN6390

ALC1001

ALC1497

SH1000

ALC2732

ALC1435

ALC1437

ALC1436

ALC2200

ALC2770

ALC1540

ALC1740

ALC1747

ALC1737

ALC1741

ALC1742

ALC1743

ALC136

ALC2322

COL

RUSA168

ISP479C

6850

Newman

MRSA 67-0

Duke strains 28, 153, 237

Plasmids

PSK236

PALC1420

PALC1421

PALC1422

\author{
Laboratory strain related to 8325-4, harboring an 11-bp deletion in rsbU \\ sigB mutant of RN 6390 \\ Complemented sigB mutant of RN6390 \\ Functional $r s b U$ derivative of $8325-4, r s b U^{+}$ \\ sarA mutant of $\mathrm{SH} 1000$ \\ RN6390 with pALC1420 (gfpurr with sarA P1 promoter) \\ RN6390 with PALC1421 (gfpurr with sarA P2 promoter) \\ RN6390 with PALC1422 (gfpurr with sarA P3 promoter) \\ RN6390 with asp23 promoter driving gfp urr in pSK236 \\ $\mathrm{SH} 1000$ with asp 23 promoter driving $g f_{\text {uvr }}$ in $\mathrm{PSK} 236$ \\ RN6390 with sarA P3-P1-P2 promoter driving $g p_{\text {uvr }}$ in PSK236 \\ gfpuvr with hla promoter in pSK236 in RN6390 \\ gfpurr with fnbA promoter in PSK236 in RN6390 \\ gfpurr with $f n 6 B$ promoter in PSK236 in RN6390 \\ gfpuvr with spa promoter in pSK236 in RN6390 \\ gfp uvr with agr RNAll promoter in pSK236 in RN6390 \\ gfpurr with agr RNAlll promoter in pSK236 in RN6390 \\ sarA mutant of RN6390 \\ FDA486 with asp23 promoter driving gfpurr in pSK236 \\ Methicillin-resistant laboratory strain \\ $\operatorname{sig} B$ mutant of $\mathrm{COL}$ \\ Spontaneous plasmid-cured derivative of parental strain ISP479 \\ Clinically isolated wild-type strain \\ Laboratory strain \\ Wound isolate \\ Isolates from patients with infective endocarditis
}

45

28

28

17

This study

22

22

22

24

24

22

This study

This study

This study

This study

This study

This study

48

This study

49

49

50

51

52

53

54

Shuttle vector containing pUC19 cloned into the HindIII site of pC194 
visible colonies were counted. As the binding patterns of the clinical and laboratory isolates were virtually identical, data were pooled within each isolate group, and bacterial binding was quantified as the percentage of the initial inoculum bound; the impact of salicylic acid exposure on binding was expressed as the mean reduction $( \pm \mathrm{SD})$ of fibrinogen or fibronectin binding when compared with that of control cells unexposed to salicylic acid, from six separate runs.

Bacterial cells were pre-exposed to $6 \mathrm{mM}$ sodium azide for 60 minutes at room temperature after overnight culture in the absence and presence of salicylic acid; then binding experiments were repeated to ascertain the requirement for an energy-dependent process.

To evaluate whether the effects of salicylic acid on $S$. aureus binding to fibrinogen and fibronectin are mediated via the upregulation of a serine protease (which cleaves and degrades such adhesins), we performed the fibronectin-binding experiments in parallel with cultures pre-exposed to $0.4 \mathrm{U}$ of the global protease inhibitor $\alpha_{2}$-macroglobulin (12).

The sarA operon positively regulates the expression of a number of adhesin genes $(13,14)$, while the global stress regulon $\operatorname{sig} B$ negatively regulates the function of several adhesin genes (via repression of sarA gene expression; refs. 15, 16). To determine whether sigB is involved in salicylic acid-mediated effects on S. aureus binding to fibrinogen and fibronectin, we evaluated fibrinogen and fibronectin binding in vitro in the following constructs: parental strain COL; its respective sigB deletion mutant; parental strain RN6390; its respective $\operatorname{sig} B$ deletion mutant; and its sigB-complemented strain. To explore the role of $r s b U$ within the sig $B$ operon, we also compared the salicylic acid-mediated effects on adhesin binding of RN6390, which carries a natural $r s b U$ deletion, with those of SH1000, a derivative of RN6390 that carries a functional $r s b U$ gene (17). To determine whether the sarA operon is involved in $S$. aureus binding to the above adhesion molecules, salicylic acid-mediated effects on S. aureus binding to fibrinogen and fibronectin were evaluated in sarA knockout mutants of RN6390 and SH1000.

Impact of salicylic acid on hemolytic and thrombolytic (platelet lysis) activity of $S$. aureus. In addition to matrix protein binding, the secretion of exotoxins, such as $\alpha$-hemolysin, represents a major virulence factor in staphylococcal pathogenesis by virtue of its cytolytic effect on many types of host cells (18). We thus evaluated whether salicylic acid would impact $\alpha$-hemolysin activity in S. aureus, using two distinct target cells for this enzyme, rabbit erythrocytes and rabbit platelets.

For hemolytic studies, cells in each tube were washed, equalized at $\mathrm{OD}_{600}$, and centrifuged at 5,000 $g$ for 10 minutes to pellet bacteria. Aliquots of serial dilutions of culture supernatants were added to a $1 \%$ suspension of washed rabbit erythrocytes in $0.01 \mathrm{M}$ PBS ( $\mathrm{pH} 7.2$ ) containing 0.1\% BSA (19). Purified $\alpha$-hemolysin $(1 \mu \mathrm{g} / \mathrm{ml}$; kindly provided by Barbara E. Menzies, Vanderbilt University, Nashville, Tennessee, USA) was used as a positive control. Since supernatants of both the parental clinical and laboratory $S$. aureus strains yielded virtually identical hemolytic activity, pooled data for each isolate group were expressed as mean units of hemolytic activity $( \pm S D)$ of all parental strains per milliliter of culture supernatant from six separate runs. The hemolytic units were defined as the reciprocal of the highest dilution of the culture supernatant that caused $50 \%$ hemolysis as measured by optical densitometry (19).

For the thrombolytic studies, washed platelets were suspended in Tyrode's medium and adjusted to an $\mathrm{OD}_{600}$ of 1.0 (equal to approximately $10^{9}$ platelets $/ \mathrm{ml}$ ). Platelets were then exposed to serial dilutions of culture supernatants. Thrombolysis was monitored by measurement of the fall in $\mathrm{OD}_{600}$ over 30 minutes (19). Purified $\alpha$-hemolysin and the membrane detergent Triton $\mathrm{X}-100(0.1 \%)$ were used as positive thrombolysis controls. Exposure of rabbit platelets to Triton X-100 or purified $\alpha$-hemolysin resulted in $100 \%$ lysis (i.e., decrease in the $\mathrm{OD}_{600}$ of the platelet suspension from 1.0 to base line) within 30 minutes. Thrombolytic activity was expressed as the mean fall of $\mathrm{OD}_{600}( \pm \mathrm{SD})$ as normalized to Triton X-100 from six separate runs.

Impact of salicylic acid on $\alpha$-bemolysin production. As a complement to the above functional assays for $\alpha$-hemolysin, we studied the impact of salicylic acid on the specific production of this toxin. Culture supernatants from equal numbers of $S$. aureus parental cells Newman and RN6390, and the relevant sigB deletion and $\operatorname{sig} B$-complemented variants, precultured in the absence or presence of salicylic acid, were diluted and applied to a $12 \%$ SDS-polyacrylamide gel for $\alpha$-hemolysin detection by immunoblot. The gel proteins were electrophoretically transferred to nitrocellulose membranes. After the membranes were blotted, they were blocked with $0.5 \%$ Tween- 20 in Trisbuffered saline, and then incubated for 1 hour with rabbit polyclonal anti- $\alpha$-hemolysin serum (1:100 dilution). The blot was developed with goat anti-rabbit IgG-alkaline phosphatase conjugate (Molecular Probes Inc., Eugene, Oregon, USA) at a 1:2,000 dilution using nitroblue tetrazolium and 5-bromo-4chloro-3-indolyl phosphate as the substrate (20).

Impact of salicylic acid on protease and lipase production. We evaluated the effects of salicylic acid on lipase and protease secretion, two sarA-repressible phenotypes, by spectrophotometric assays in postexponential culture supernatants of the parental study strains, Newman, RN6390, COL, and ISP479C, as well as in the sigB mutant and sigB-complemented variant of RN6390, in the presence or absence of salicylic acid $(50 \mu \mathrm{g} / \mathrm{ml})$.

For quantification of global protease activity, the azocasein spectrophotometric hydrolysis assay was used. After equalizing all cultures at $\mathrm{OD}_{600}, 300 \mu \mathrm{l}$ of culture supernatants were added to $800 \mu \mathrm{l}$ of an azocasein solution and incubated for 16 hours at $37^{\circ} \mathrm{C}$. Undegraded azocasein was precipitated by the addition of $400 \mu \mathrm{l}$ of $50 \%$ trichloroacetic acid and removed 
by centrifugation. Protease activity was indicated by clearing of the supernatant solution with a concomitant decrease in $\mathrm{OD}_{340}(21)$.

For quantification of lipase activity, a tributyrin assay was used, as previously described (21). In brief, $1 \mathrm{ml}$ of the prewarmed tributyrin emulsion $\left(50^{\circ} \mathrm{C}\right)$ was added to $100 \mu \mathrm{l}$ of each standardized culture supernatant. The OD was monitored at $450 \mathrm{~nm}$ at room temperature. Lipase activity was indicated by a clearing of the emulsion and quantified by a subsequent decrease in OD.

As the protease and lipase activities of parental $S$. aureus cells were virtually identical, data were pooled, and the impact of salicylic acid was expressed as the result of six separate runs based on the normalized OD of cells grown in the absence of salicylic acid.

Whole-cell ELISA for detection of cell wall-associated protein A. To determine levels of protein A production (a sarA-repressible phenotype), cell wall-associated proteins were extracted from overnight cultures of strain Newman and of strain RN6390, its sigB mutant, and its sigB-complemented strain, using lysostaphin in a hypertonic medium (30\% raffinose). Microtiter wells were coated with human IgG at $1 \mu \mathrm{g} / \mathrm{ml}$ overnight at $37^{\circ} \mathrm{C}$. Serial dilutions of extracts of cell wall proteins in $1 \times$ PBS, $0.05 \%$ Tween- 20 , and a control protein A standard (Calbiochem Corp., San Diego, California, USA) were added to microtiter wells, then probed with affinity-purified chicken anti-protein A antibody (Accurate Chemical \& Scientific Group., Westbury, New York, USA) at a 1:3,000 dilution. Bound antibody was detected by immune colorimetry with rabbit antichicken $\operatorname{IgG}$ (1:5,000 dilution) conjugated to alkaline phosphatase (Jackson ImmunoResearch Laboratories Inc., West Grove, Pennsylvania, USA), using $p$-nitrophenyl phosphate as a developing substrate. Microtiter wells were then read in an ELISA plate reader (Bio-Tek Instruments Inc., Winooski, Vermont, USA) and expressed as the average of two runs.

Impact of salicylic acid on promoter gene expression in vitro and in vivo. To define whether salicylic acid-induced changes in $S$. aureus binding to matrix proteins, and $\alpha$-hemolysin production, were attributable to impacts on virulence gene expression, we employed a gene reporter system. We used a shuttle plasmid with a promoterless, modified GFP reporter gene ( $g f_{w}$ wr $)$ that has been optimized for use in fluorometry and flow cytometry analyses $(22,23)$. The following promoters relevant to the present study were cloned upstream of the gfp wor reporter gene in strains RN6390, SH1000, and FDA486 to assay the impact of salicylic acid as previously detailed (23): $f n b A$ and $f n b B$ promoters (the two major fibronectin protein-binding genes); bla promoter; spa promoter; individual sarA $\mathrm{P} 1$ and sarA $\mathrm{P} 3$ promoters; sarA P2-P3-P1 promoter; agr RNAII and RNAIII promoters; and asp23 promoter (a sigB-dependent locus, and a surrogate for $\operatorname{sig} B$ promoter activation; ref. 24). The GFP excitation maximum in these constructs has been shifted from 395 to $488 \mathrm{~nm}$ by a single substitution at residue 65 (serine to threonine [S65T]), yielding
$\operatorname{GFP}_{w r}(22,23)$. GFP fluorescence was detected by excitation at $488 \mathrm{~nm}$ and emission at $520 \mathrm{~nm}$. Fluorometry (Turner Fluorometer model 450; Barnstead International, Dubuque, Iowa, USA) or flow cytometry (FACSCalibur; Becton Dickinson Biosciences, Sparks, Maryland, USA) was conducted on equal concentrations of bacterial cells to quantitate in vitro expression of GFP. Results were expressed as the average of three separate runs. Fluorescence microscopy (Olympus America Inc., Melville, New York, USA) was used to detect the impact of acetylsalicylic acid or salicylic acid on in vivo expression of GFP in cells within vegetative lesions removed from rabbits with infective endocarditis (22). The slides were also stained with Giemsa stain for localization of bacteria within the vegetations. In addition, to provide a more quantitative assessment of in vivo gene expression, 10,000 intravegetation staphylococcal cells from control or aspirin-treated rabbits were evaluated for sarA P1 GFP fluorescence by flow cytometry as previously described (25).

Impact of salicylic acid on sarA gene transcription by Northern analysis. For staphylococcal RNA extraction, 10-ml cultures of S. aureus RN6390 and Newman were grown to mid-log phase $\left(\mathrm{OD}_{650}, 0.7\right)$, late log phase $\left(\mathrm{OD}_{650}\right.$, $1.1)$, and postexponential phase $\left(\mathrm{OD}_{650}, 1.7\right)$, pelleted by centrifugation, resuspended in FastRNA reagent (Qbiogene Inc., Carlsbad, California, USA) in combination with 0.1-mm Zirconia-silica beads, and shaken at 6,000 $\mathrm{rpm}$ in a high-speed homogenizer for 20 seconds. After addition of $200 \mu \mathrm{l}$ chloroform, the sample was centrifuged at $12,000 \mathrm{~g}$ at $4^{\circ} \mathrm{C}$ for 10 minutes. The RNA located in the upper aqueous layer was precipitated with isopropanol and resuspended in water treated with Diethyl pyrocarbonate. The integrity of the RNA species was verified with a formaldehyde gel (26). Ten micrograms of RNA from each culture condition was electrophoresed through a $1.5 \%$ agarose, $0.66 \mathrm{M}$ formaldehyde gel in Mops running buffer (26). RNA was blotted onto Hybond $\mathrm{N}^{+}$membranes (Amersham Biosciences Corp., Piscataway, New Jersey, USA) using a TurboBlotter alkaline transfer system (Schleicher $\&$ Schuell Inc., Keene, New Hampshire, USA). For detection of sarA transcripts, gel-purified DNA probes were radiolabeled with $\left[\alpha-{ }^{32} \mathrm{P}\right] \mathrm{dCTP}$ by the random-primer method, and hybridized under high-stringency conditions. The blots were subsequently washed and autoradiographed (27).

Impact of salicylic acid on SarA protein production. S. aureus strain SH1000, carrying an intact sigB operon, was diluted 1:100 from an overnight culture into $50 \mathrm{ml}$ of tryptic soy broth containing $50 \mu \mathrm{g} / \mathrm{ml}$ of salicylic acid; the control culture did not contain any salicylic acid. Both cultures grew to similar OD and were harvested 8 hours later (the time of maximal SarA protein expression). Cell-free extracts of each culture were prepared as described (28). The protein concentration of each preparation was determined by the Bradford assay (Bio-Rad Laboratories Inc., Hercules, California, USA). Equivalent amounts of cell-free extract of each cell preparation were then loaded onto a $12 \%$ SDS gel, resolved, and blotted 
Table 2

Effect of salicylic acid on fibronectin and fibrinogen binding, hemolysis, and thrombolysis of laboratory S. aureus strains and clinical S. aureus isolates

\begin{tabular}{lcccc}
\hline & \multicolumn{2}{c}{ Laboratory isolates } & \multicolumn{2}{c}{ Clinical isolates } \\
& $\mathrm{SAL}, 30 \mu \mathrm{g} / \mathrm{ml}$ & $\mathrm{SAL}, 50 \mu \mathrm{g} / \mathrm{ml}$ & $\mathrm{SAL}, 30 \mu \mathrm{g} / \mathrm{ml}$ & $\mathrm{SAL}, 50 \mu \mathrm{g} / \mathrm{ml}$ \\
Fibronectin binding & $-35.9 \% \pm 6.5 \%^{\mathrm{A}}$ & $-54.5 \% \pm 5.1 \%^{\mathrm{B}}$ & $-28.9 \% \pm 3.5 \%^{\mathrm{B}}$ & $-51.4 \% \pm 2.0 \%^{\mathrm{B}}$ \\
Fibrinogen binding & $-35.4 \% \pm 5.2 \%^{\mathrm{A}}$ & $-55.1 \% \pm 4.9 \%^{\mathrm{B}}$ & $-32.4 \% \pm 2.1 \%^{\mathrm{B}}$ & $-53.4 \% \pm 2.9 \%^{\mathrm{B}}$ \\
Hemolysis & $-48 \% \pm 4 \%^{\mathrm{B}}$ & $-62 \% \pm 5 \%^{\mathrm{B}}$ & $-33 \% \pm 13 \%^{\mathrm{A}}$ & $-54 \% \pm 12 \%^{\mathrm{B}}$ \\
Thrombolysis & $-32 \% \pm 4 \%^{\mathrm{A}}$ & $-45 \% \pm 2 \%^{\mathrm{B}}$ & $-30 \% \pm 6 \%^{\mathrm{A}}$ & $-48 \% \pm 9 \%^{\mathrm{B}}$
\end{tabular}

Numbers listed in the table indicate the mean reduction $( \pm S D)$ as compared with controls (S. aureus precultured in plain medium) used as the standard (i.e., 100\%). Binding of S. aureus control cells (laboratory or clinical isolates) to fibrinogen or fibronectin ranged from $7.1 \%$ to $10.2 \%$ of the inoculum. Hemolytic and thrombolytic activity of $S$. aureus control cells ranged from 550 to 800 hemolytic units and from $56 \%$ to $84 \%$, respectively. The reductions in matrix protein binding and lysis represent composite pooled data derived from the laboratory S. aureus strains RN6390, COL, Newman, and ISP479C, and the clinical S. aureus isolates MRSA 67-0, 6850, Duke 28, Duke 153, and Duke 237. SAL, salicylic acid. ${ }^{A} P<0.002,{ }^{B} P<0.001$.

onto nitrocellulose. The blot was then incubated with blocking buffer, probed with an anti-SarA murine $\mathrm{mAb}$ (1:1,000 dilution) followed by a sheep anti-mouse-alkaline phosphatase conjugate (1:5,000 dilution) (Jackson ImmunoResearch Laboratories Inc.), and then developed as previously described (28). Band intensities were then quantitated by densitometry using SigmaGel software (SPSS Inc., Chicago, Illinois, USA) and expressed as the average of two separate experiments.

Rabbit endocarditis model. To evaluate the in vivo role of the $\operatorname{sar} A$ and the $\operatorname{sig} B$ operons in mediating the antimicrobial effects of salicylic acid, we induced endocarditis in rabbits with the parental strain RN6390 and its $r s b U$ repaired derivative SH1000; the parental strain COL; the respective $\operatorname{sig} B$ and $s a r A$ mutants; and the sigB-complemented variant of RN6390. In brief, anesthetized rabbits (New Zealand white; Irish Farms, Corona, California, USA) underwent transcarotid-transaortic valve catheterization with a polyethylene catheter to induce sterile aortic valve vegetations. The catheter remained indwelling for the duration of this study. Animals were challenged intravenously with the $\mathrm{ID}_{95}$ inoculum of the study S. aureus strains (as established by pilot studies) at 24 hours after catheterization. Oral treatment (by gavage) with $8 \mathrm{mg} / \mathrm{kg} / \mathrm{d}$ salicylic acid or intravenous treatment with $8 \mathrm{mg} / \mathrm{kg} / \mathrm{d}$ acetylsalicylic acid (Bayer AG, Leverkusen, Germany) was initiated at 24 hours after infection, over the next 72 hours. This dose was previously established (8) to exert maximal antimicrobial effects against $S$. aureus in this model. At 96 hours after infection, animals were euthanized with a rapid intravenous injection of $100 \mathrm{mg} / \mathrm{kg}$ sodium pentobarbital (Abbott Laboratories, Chicago, Illinois, USA). During autopsy, vegetations and kidneys were removed to determine vegetation weights, as well as bacterial densities in vegetations and kidney lesions (8). In addition, vegetations were processed for in vivo fluorescence microscopy as previously described (22). All rabbits were treated in accordance with institutional and US Public Health Service guidelines for the humane care and treatment of animals.
Statistics. For in vitro experiments, the one-way Kruskal-Wallis ANOVA test and the Dunn's post hoc comparison were used to compare data sets generated for $S$. aureus cells precultured in salicylic acid $(30$ or $50 \mu \mathrm{g} / \mathrm{ml}$ ) with those for untreated control cells. When control cells were compared with cells precultured in a single dose of salicylic acid, the two-sided MannWhitney $U$ test was used. For in vivo experiments, the two-sided Mann-Whitney $U$ test was used to compare data sets generated in salicylic acid-treated and untreated animals. A $P$ value $\leq 0.05$ was considered significant.

\section{Results}

In vitro effect of salicylic acid on S. aureus virulence phenotypes upregulated by sarA. Parental S. aureus cells precultured in the presence of salicylic acid demonstrated a substantial, dose-dependent reduction in binding to both fibronectin and fibrinogen, at two clinically achievable serum concentrations (Table 2). The mitigating effects of salicylic acid on $S$. aureus binding to these matrix proteins were virtually eliminated when the organism was pre-exposed to sodium azide, an inhibitor of oxidative phosphorylation (data not shown); this emphasized the requirement for an energy- dependent process in viable bacteria to fully execute this salicylic acid-mediated effect. Similar to the extent of binding, the extents of hemolysis and thrombolysis, presumably mediated by $\alpha$-hemolysin in supernatants obtained from cells precultured in the presence of salicylic acid, were significantly less than observed with control cells. The extent of mitigation of matrix protein binding and target cell cytolysis was very similar between clinical and laboratory strains.

The in vitro hemolytic, thrombolytic (platelet lysis), and matrix protein-binding assays were repeated with two other clinically available NSAIDs, aspirin (the parent compound of salicylic acid) and indomethacin. Neither of these agents yielded any substantial impact on the cytolytic or matrix-binding phenotypes in parental strains RN6390, COL, or Newman (data not shown). Thus, there was a relative specificity of salicylic acid as compared with several clinically used NSAIDs in regard to the above impacts on the $S$. aureus phenotypes examined. More importantly, these data substantiated the requirement of the conversion of acetylsalicylic acid to salicylic acid for its in vitro antimicrobial properties.

In vitro effect of salicylic acid on S. aureus phenotypic and genotypic parameters downregulated by sarA. The downregulation in the expression of fibronectin-binding proteins and $\alpha$-hemolysin due to salicylic acid is consistent with a downregulation in SarA-mediated effects. To assess other phenotypes that might be upregulated in $S$. aureus as a result of diminished SarA expression, we examined 
Table 3

Effect of $50 \mu \mathrm{g} / \mathrm{ml}$ salicylic acid on fibronectin and fibrinogen binding, hemolysis, and thrombolysis of the S. aureus strains listed

\begin{tabular}{|c|c|c|c|c|c|c|c|c|}
\hline & $\begin{array}{l}\text { RN6390 } \\
\text { parent }\end{array}$ & $\begin{array}{c}\text { RN6390 } \\
\text { sarA } A^{-} \text {mutant }\end{array}$ & $\begin{array}{c}\mathrm{SH} 1000 r^{\mathrm{S} b U^{+}} \\
\text {derivative }\end{array}$ & $\begin{array}{c}\mathrm{SH} 1000 \\
\text { sarA- mutant }\end{array}$ & $\begin{array}{c}\text { RN6390 } \\
\text { sigB mutant }\end{array}$ & $\begin{array}{c}\text { RN6390 } \\
\text { sigB compl. }\end{array}$ & $\begin{array}{c}\mathrm{COL} \\
\text { parent }\end{array}$ & $\begin{array}{c}\mathrm{COL} \operatorname{sig} B \\
\text { mutant }\end{array}$ \\
\hline Fibronectin binding & $-54.6 \pm 7.3^{\mathrm{A}}$ & $-14.38 \pm 3.4$ & $-40.8 \pm 3.6^{A}$ & $-7.2 \pm 0.4$ & $-8.6 \pm 2.6$ & $-49.7 \pm 6.2^{\mathrm{A}}$ & $-53.6 \pm 6.1^{\mathrm{A}}$ & $-5.6 \pm 0.7$ \\
\hline Fibrinogen binding & $-51.6 \pm 5.2^{\mathrm{A}}$ & $-11.16 \pm 4.1$ & $-45.6 \pm 3.7^{\mathrm{A}}$ & $-12.6 \pm 0.8$ & $-7.1 \pm 2.2$ & $-51.2 \pm 6.5^{\mathrm{A}}$ & $-51.2 \pm 6.9^{\mathrm{A}}$ & $-6.6 \pm 1.0$ \\
\hline Hemolysis & $-64 \pm 8^{A}$ & $-9 \pm 2$ & $-51 \pm 5^{\mathrm{A}}$ & $-10 \pm 14$ & $-4 \pm 1$ & $-56 \pm 9^{A}$ & $-67 \pm 5^{A}$ & $-3 \pm 1$ \\
\hline Thrombolysis & $-58 \pm 7^{A}$ & $-13 \pm 2$ & $-45 \pm 6^{A}$ & $-6 \pm 4$ & $-3 \pm 1$ & $-53 \pm 6^{A}$ & $-49 \pm 4^{\mathrm{A}}$ & $-2 \pm 1$ \\
\hline
\end{tabular}

The background of each strain is indicated next to the mutant type. Numbers indicate the percent mean reduction $( \pm$ SD) as compared with controls $(S$. aureus precultured in plain medium). Compl., complemented. ${ }^{A} P<0.003$.

protease and lipase activities. Protease and lipase activities were elevated in supernatants from parental S. aureus strains exposed to salicylic acid: protease activity was increased fivefold $(P<0.0001)$ and lipase activity was increased 2.6-fold $(P<0.0001)$ in supernatants from salicylic acid-treated cells versus untreated controls. The salicylic acid-induced increase of protease activity was blunted in cells precultured in the presence of the global protease inhibitor $\alpha_{2}$-macroglobulin. However, 50 $\mu \mathrm{g} / \mathrm{ml}$ salicylic acid caused similar and significant reductions in fibronectin binding of the above parental strains in the presence or absence of pre-exposure to $\alpha_{2}$-macroglobulin $(45.7 \% \pm 4.2 \%$ vs. $53.9 \% \pm 5.5 \%$ reductions, respectively; $P<0.0001)$. These findings provide evidence that the salicylic acid-induced suppression of the $S$. aureus fibronectin-binding phenotype is not principally related to an increase in protease secretion that leads to adhesin inactivation (12).

A mean increase of $17.6 \% \pm 3.9 \%$ in protein A levels versus untreated controls was observed in parental $S$. aureus strains exposed to $50 \mu \mathrm{g} / \mathrm{ml}$ salicylic acid. In parallel, a corresponding twofold increase in spa promoter expression was noted in salicylic acid-treated cells as compared with untreated controls, suggesting a salicylic acid-mediated impact on the structural gene and/or the respective regulator gene.

The role of sarA and sigB in salicylic acid-mediated modifications of S. aureus phenotypes. In sarA mutants of strain RN6390 and its isogenic rsbU-repaired derivative SH1000, intrinsic binding to fibrinogen and fibronectin was reduced when compared with that in the corresponding parental strains (fibrinogen, $-52.7 \% \pm 2.3 \%$; fibronectin, $-54.2 \% \pm 2.5 \%$ ). Similarly, in these same sarA mutants hemolysis and thrombolysis were reduced by $55 \% \pm 5 \%$ and $57 \% \pm 5 \%$, respectively, when compared with the parental strains. No significant reductions of matrix protein binding and cytolytic profiles were observed when the sarA mutants were pre-exposed to salicylic acid (Table 3 ). These findings confirmed the key importance of sarA in regulating staphylococcal matrix protein binding and cytolysis gene products and suggested a pivotal role for sarA in mediating the impact of salicylic acid on $S$. aureus virulence phenotypes.

In the sigB knockouts of strains RN6390 and COL, intrinsic binding to fibrinogen and fibronectin was increased when compared with that in the respective parental strains (fibrinogen, $19.4 \% \pm 3.1 \%$; fibronectin, $22.7 \% \pm 2.6 \%$ ). Hemolysis and thrombolysis in these sigB knockouts were increased by $14.6 \% \pm 5.7 \%$ and $17.5 \% \pm 4.4 \%$, respectively, when compared with those in the parental strains. Salicylic acid-mediated reductions in matrix protein binding, hemolysis, and thrombolysis observed in these parental strains were mitigated in the respective $\operatorname{sig} B$ knockouts (Table 3 ). Moreover, no impact of salicylic acid on protease-activity or lipaseactivity profiles or cell wall protein A production was detectable in the sigB knockout of RN6390. These phenotypic response parameters were restored to parental levels with complementation of sigB in the RN6390 background. Notably, salicylic acid caused similar extents of reduction in matrix protein binding and cytolytic profiles in parental strain RN6390 and its rsbU-repaired derivative SH1000 (Table 3).

In summary, the above observations suggest that in parental S. aureus strains, salicylic acid suppresses exotoxin production and matrix protein binding, while enhancing exoenzyme (e.g., protease and lipase) and protein A production. The presence of an intact sarA locus and the $s i g B$ stress operon (with or without a functional $r s b U$ locus) appears to be a prerequisite in this context. Therefore, this composite phenotype is consistent with suppression of sarA expression following sigB activation in either an $r s b U$ dependent or an $r s b U$-independent manner (13-16).

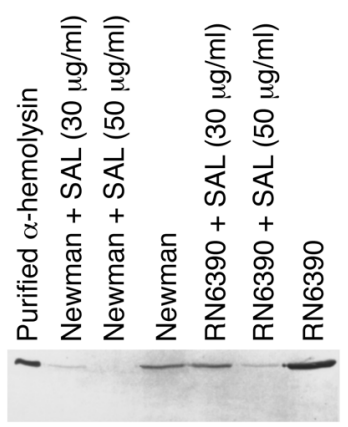

\section{Figure 1}

Western blot for detection of $\alpha$-hemolysin. An immunoblot of culture supernatants of the indicated $S$. aureus strains precultured in the absence (controls) or presence of 30 and $50 \mu \mathrm{g} / \mathrm{ml}$ salicylic acid to the early stationary phase ( 18 hours) was probed for $\alpha$-hemolysin. Affinity-purified rabbit polyclonal anti- $\alpha$-hemolysin IgG was used as the primary antibody. SAL, salicylic acid. 


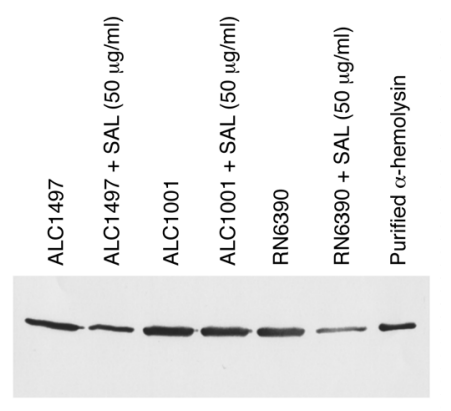

Figure 2

Western blot for detection of $\alpha$-hemolysin in isogenic strain RN6390, its $\operatorname{sig} B$ transposon mutant, and its sigB-complemented variant. An immunoblot of stationary-phase culture supernatants (18 hours) of the indicated strains precultured in the absence (controls) or presence of $50 \mu \mathrm{g} / \mathrm{ml}$ salicylic acid is shown. Affinity-purified polyclonal rabbit anti- $\alpha$-hemolysin $\lg G$ served as the primary antibody.

$\alpha$-Hemolysin production. Paralleling the hemolytic and thrombolytic data sets above, $\alpha$-hemolysin was detected by immunoblots in supernatants of parental $S$. aureus strains grown in the absence of salicylic acid but was substantially diminished in the presence of salicylic acid (Figure 1). Immunoblots of extracellular proteins derived from supernatants of postexponential-phase cultures of the RN6390 parent, its sigB mutant, and its sigB-complemented variant grown in salicylic acid disclosed a substantial reduction in $\alpha$-hemolysin production in the parental and sigB-complemented strains, as compared with little impact on $\alpha$-hemolysin production in the sigB deletion mutant (Figure 2). These data, combined with the phenotypic findings above, clearly support the notion that the $\operatorname{sig} B$ operon is pivotal in mediating the antimicrobial effects of salicylic acid in vitro in the context of cytolysis.
Salicylic acid-induced impacts on bla, fnbA, and fnbB promoter expression in vitro. In parental strain RN6390, fluorometric analyses revealed a 2.9 -fold and a 2.1 -fold decrease, respectively, in $f n b A$ and $f n b B$ promoter expression in cells treated with $50 \mu \mathrm{g} / \mathrm{ml}$ salicylic acid, as compared with untreated controls. Similarly, there was a 3.3fold reduction in bla promoter expression in salicylic acid-treated cells, compared with untreated control cells. These data demonstrate that the mitigating effects of salicylic acid on staphylococcal fibronectin binding and on $\alpha$-hemolysin production were correlated with reduced transcription of the relevant gene promoters in S. aureus.

Salicylic acid-induced impacts on sarA and agr promoter expression and sigB gene expression in vitro. By fluorometric analysis, treatment of $S$. aureus RN6390 with 50 $\mu \mathrm{g} / \mathrm{ml}$ salicylic acid caused (a) a 2.3-fold reduction in the expression of the sarA $\mathrm{P} 1$ promoter, the strongest promoter within the triple-sarA-promoter system; (b) a 1.8-fold reduction in the native triple-sarA-promoter complex (i.e., sarA P2-P3-P1); and (c) a 2.0-fold and a 2.2-fold reduction in agr RNAII and agr RNAIII promoter expression, respectively, as compared with those in untreated controls.

With respect to the sarA operon, we independently confirmed the above fluorometric data by both flow cytometry and Northern analysis. There are three promoters (P2, P3, and P1) upstream of the sarA coding region, with the proximal P1 promoter being the strongest. Flow cytometry revealed a 2.5 -fold decrease in sarA P1 promoter expression in the presence of salicylic acid, whereas the activity of the middle sarA promoter, $\mathrm{P} 3$, which is sigB-dependent, was twofold higher in salicylic acid-treated than in untreated cells (Figure 3a). Consistent with the flow cytometry data sets, Northern analysis of strains RN6390 (Figure 3b) and Newman (not shown) a

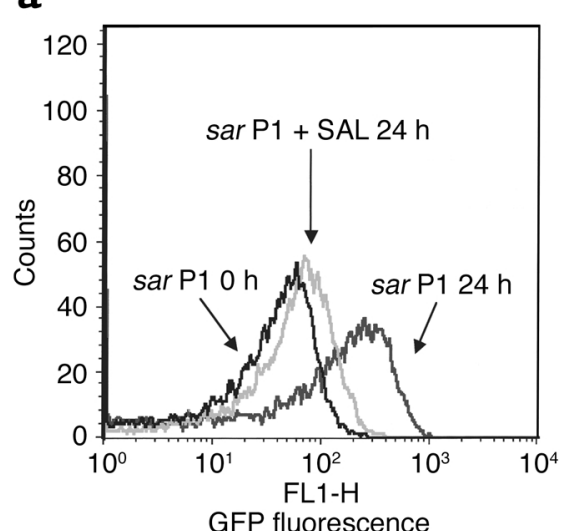

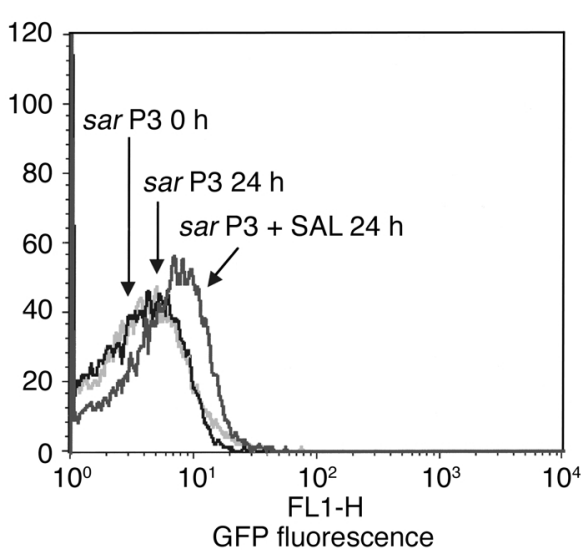

b

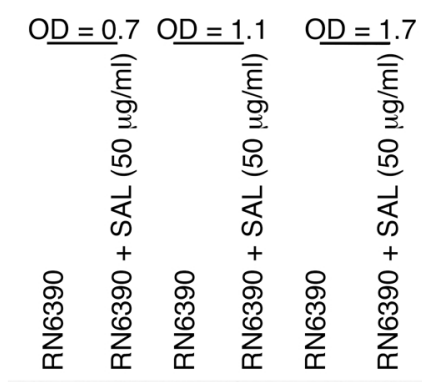

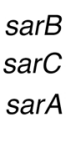

$\operatorname{sar} B$

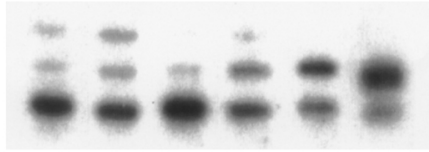

Figure 3

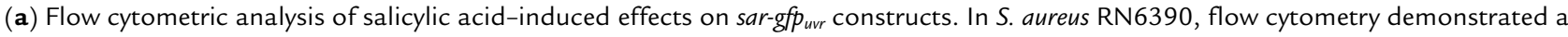
2.5-fold attenuation of P1-initiated sarA promoter activity (left) and a 1.8-fold increase of P3-initiated sarA promoter activity (right) in cultures pre-exposed to salicylic acid $(50 \mu \mathrm{g} / \mathrm{ml})$ as compared with expression in plain medium. (b) Effect of salicylic acid on sar promoter transcription in S. aureus. Northern analysis revealed that in S. aureus RN6390 cells precultured in the presence of $50 \mu g / m l$ salicylic acid, P1-initiated sarA transcription was suppressed, whereas P3-initiated sarA transcription (corresponds to sarC) was enhanced in the late log phase and postexponential phase, as compared with cells precultured in plain medium. The P2-initiated sar $A$ transcription level (corresponds to $\operatorname{sar} B$ ) was too weak to define the impact of salicylic acid on this locus. 
Table 4

Effect of salicylic acid on microbial proliferation and hematogenous dissemination in rabbits with infective endocarditis challenged with parental strain RN6390 or its sarA mutant

\begin{tabular}{|c|c|c|c|c|c|c|c|c|}
\hline \multirow[t]{2}{*}{ Strains } & \multicolumn{2}{|c|}{ Number of animals ${ }^{A}$} & \multicolumn{2}{|c|}{$\begin{array}{l}\text { Vegetation weight } \\
\text { (g) }\end{array}$} & \multicolumn{2}{|c|}{$\begin{array}{l}\text { Vegetation bacterial density } \\
\left(\log _{10} \text { CFUs/g tissue }\right)\end{array}$} & \multicolumn{2}{|c|}{$\begin{array}{l}\text { Renal bacterial density } \\
\left(\log _{10} \text { CFUs/g tissue) }\right.\end{array}$} \\
\hline & Contr & Treatment & Control & Treatment & Control & Treatment & Control & Treatment \\
\hline RN6390 & 8 & 8 & $0.12 \pm 0.01$ & $0.07 \pm 0.01^{B}$ & $8.32 \pm 0.93$ & $6.19 \pm 0.92^{B}$ & $8.41 \pm 0.88$ & $6.19 \pm 0.89^{\mathrm{E}}$ \\
\hline sarA mutant & 7 & 8 & $0.12 \pm 0.01$ & $0.11 \pm 0.02$ & $7.56 \pm 0.63$ & $7.28 \pm 1.43$ & $7.49 \pm 0.65$ & $7.34 \pm 1.37$ \\
\hline
\end{tabular}

In vivo plasma levels of salicylic acid were evaluated in the treatment groups by an immunoassay and were $51 \pm 2.9 \mu \mathrm{g} / \mathrm{ml}(47-55 \mu \mathrm{g} / \mathrm{ml})$. ${ }^{\mathrm{A}} \mathrm{Mean} \mathrm{mortality}$ rate $13.9 \%$ (range 11.1-22.2\%). Mean mortality rate without significant differences between groups. ${ }^{\mathrm{B} P}<0.007$ vs. control.

demonstrated that the sarA P3 transcript level was increased in cells pre-exposed to salicylic acid when compared with untreated cells, while the sarA P1 transcript level was decreased compared with that in untreated cells.

The upregulation of the sarA P3 transcript in response to salicylic acid further suggested a salicylic acid-induced upregulation of $\operatorname{sig} B$. Therefore, we used fluorometry to analyze the impact of salicylic acid on expression of a major $\operatorname{sig} B$-dependent promoter, asp23, in three $S$. aureus strains with two distinct backgrounds: (a) RN6390, carrying a natural $r s b U$ deletion within the sigB operon; (b) SH1000, the functional $r s b U$ derivative of RN6390; and (c) FDA486, carrying an intact $\operatorname{sig} B$ operon. In these strains, salicylic acid caused a 1.5- to 1.9-fold increase in asp23 promoter expression as compared with that in untreated cells. Collectively, these data demonstrate a net salicylic acid-mediated downmodulation of both the sarA and the agr operons, associated with an upregulation of $\operatorname{sig} B$, which occurred in both the presence and the absence of an intact $r s b U$ locus within the $\operatorname{sig} B$ operon.

Expression of SarA protein in S. aureus strain SH1000 upon exposure to salicylic acid. To determine whether a change in SarA protein level occurred in strain SH1000 (with an intact sigB locus) when it was exposed to salicylic acid, we probed an immunoblot containing equivalent amounts of early stationary-phase cell extracts of strain SH1000, precultured in the presence and absence of salicylic acid, with an anti-SarA mAb. At $50 \mu \mathrm{g}$ of cellular proteins, there was a $10 \%$ reduction in SarA protein levels in cells precultured in salicylic acid at $50 \mu \mathrm{g} / \mathrm{ml}$ (516 vs. 576 densitometric units in treated and untreated cells, respectively).

The role of the sarA locus in salicylic acid-mediated effects in vivo. Aortic-catheterized rabbits were challenged intravenously with either parental strain RN6390 or its isogenic sarA knockout mutant. Rabbits treated with 8 $\mathrm{mg} / \mathrm{kg} / \mathrm{d}$ salicylic acid and infected with parental strain RN6390 demonstrated significantly smaller vegetations and lower bacterial densities in vegetations and kidney lesions than did their respective untreated controls; these effects were substantially blunted in salicylic acid-treated rabbits challenged with the sarA knockout mutant (Table 4). Thus, the sarA locus plays a predominant role in mediating the in vivo effects of salicylic acid, paralleling the in vitro data above.
To further substantiate the in vivo downmodulatory impact of salicylic acid on sarA expression, aorticcatheterized rabbits and infected with the $S$. aureus RN6390 sarA P1:gfpur construct either went untreated or received $8 \mathrm{mg} / \mathrm{kg} / \mathrm{d}$ aspirin intravenously. While bacterial cells in vegetations from control animals demonstrated a high level of in situ GFP fluorescence, cells from aspirin-treated rabbits showed a substantial reduction in GFP fluorescence, indicative of reduced sarA P1 expression in the presence of salicylic acid in vivo
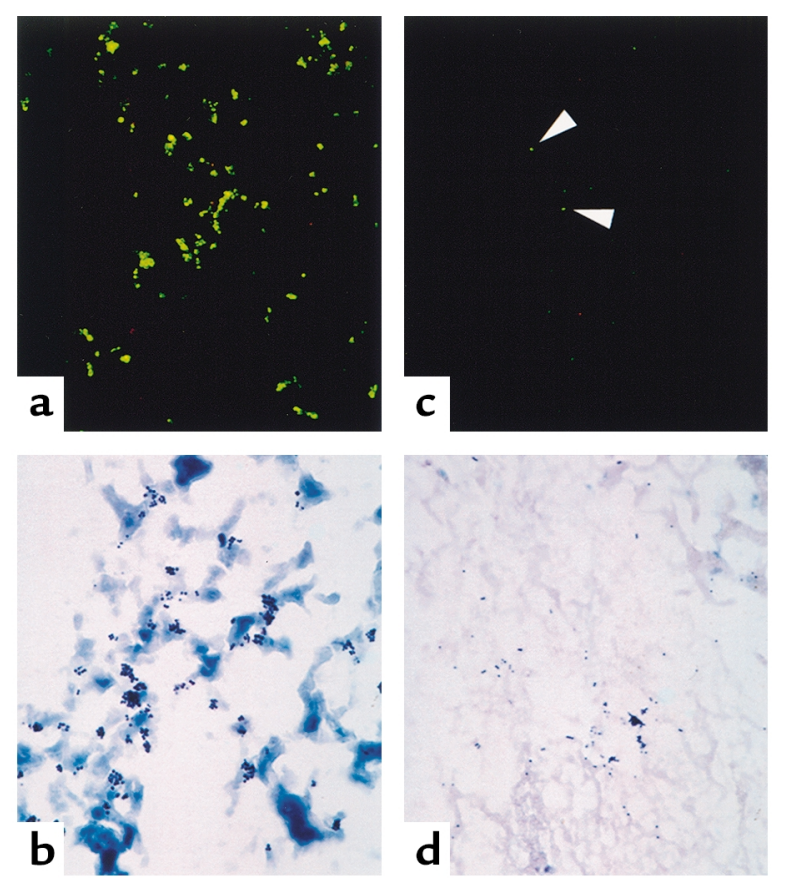

\section{Figure 4}

Fluorescence analysis of aspirin-induced effects on sarA P1:gfpuvr constructs in vivo. Vegetative lesions were removed from rabbits with infective endocarditis 24 hours after they received no treatment (controls) or a single intravenous dose of $8 \mathrm{mg} / \mathrm{kg}$ aspirin, and fluorescence microscopy was conducted on sarA P1:gfpur constructs within the lesions. In vegetations from control rabbits, bright green fluorescence (a) can be clearly detected as originating from the respective cells shown in the adjacent section stained with Giemsa (b). In comparison, the fluorescence intensities of individual bacterial cells found in Giemsa-stained vegetation sections of aspirin-treated rabbits (d) show a marked reduction in fluorescence (c, arrowheads), even at the highest immunofluorescent microscopy gain (magnification $\times 350$ ). 
Table 5

Effect of salicylic acid on microbial proliferation in vegetative lesions and hematogenous dissemination to kidneys of rabbits with experimental endocarditis due to the $S$. aureus strains listed

\begin{tabular}{|c|c|c|c|c|c|c|c|c|}
\hline \multirow[t]{2}{*}{ Strains } & \multicolumn{2}{|c|}{ Number of animals ${ }^{A}$} & \multicolumn{2}{|c|}{$\begin{array}{l}\text { Vegetation weight } \\
\text { (g) }\end{array}$} & \multicolumn{2}{|c|}{$\begin{array}{l}\text { Vegetation bacterial density } \\
\quad\left(\log _{10} \text { CFUs/g tissue }\right)\end{array}$} & \multicolumn{2}{|c|}{$\begin{array}{l}\text { Renal bacterial density } \\
\left(\log _{10} \text { CFUs/g tissue) }\right.\end{array}$} \\
\hline & Control & Treatment & Control & Treatment & Control & Treatment & Control & Treatment \\
\hline RN6390 & 7 & 6 & $0.12 \pm 0.01$ & $0.07 \pm 0.01^{\mathrm{B}}$ & $8.42 \pm 0.79$ & $6.45 \pm 0.91^{c}$ & $8.05 \pm 0.89$ & $6.38 \pm 0.9 c$ \\
\hline sigB mutant (RN6390) & 6 & 6 & $0.13 \pm 0.01$ & $0.12 \pm 0.01$ & $8.71 \pm 0.89$ & $8.37 \pm 0.99$ & $8.47 \pm 0.79$ & $8.17 \pm 0.94$ \\
\hline sigB complemented (RN6390) & 6 & 6 & $0.11 \pm 0.01$ & $0.06 \pm 0.01^{B}$ & $7.97 \pm 0.78$ & $5.98 \pm 0.66^{C}$ & $7.95 \pm 0.86$ & $6.09 \pm 0.96 \mathrm{C}$ \\
\hline SH1000 & 5 & 5 & $0.25 \pm 0.07$ & $0.18 \pm 0.06$ & $6.54 \pm 0.58$ & $4.83 \pm 0.7^{\mathrm{E}}$ & $5.39 \pm 1.14$ & $3.43 \pm 0.8^{\mathrm{E}}$ \\
\hline $\mathrm{COL}$ & 7 & 7 & $0.17 \pm 0.09$ & $0.05 \pm 0.02^{\mathrm{B}}$ & $8.27 \pm 0.92$ & $5.1 \pm 0.77 \mathrm{C}$ & $6.62 \pm 1.02$ & $4.08 \pm 0.82^{\mathrm{D}}$ \\
\hline $\operatorname{sig} B$ mutant $(\mathrm{COL})$ & 6 & 7 & $0.13 \pm 0.04$ & $0.12 \pm 0.06$ & $7.24 \pm 0.61$ & $7.2 \pm 0.68$ & $6.44 \pm 0.79$ & $6.36 \pm 1.25$ \\
\hline
\end{tabular}

Each respective background strain is indicated next to the mutant. In vivo plasma levels of salicylic acid were evaluated in the treatment groups by an immunoassay and were $49 \pm 2.4 \mu \mathrm{g} / \mathrm{ml}(46-57 \mu \mathrm{g} / \mathrm{ml})$. ${ }^{A}$ Mean mortality rate $8.4 \%$ (range $0-14.3 \%$ ). Mean mortality rate without significant differences between groups. ${ }^{\mathrm{B}} P<0.002$ vs. control group; ${ }^{C} P<0.01$ vs. control group; ${ }^{\mathrm{D}} P<0.03$ vs. control group; ${ }^{\mathrm{E}} P<0.05$ vs. control group.

(Figure 4). These observations were quantitatively confirmed via flow cytometry by the substantially reduced sarA P1 promoter-driven GFP expression among 10,000 staphylococcal cells isolated from aspirin-treated versus control vegetations (data not shown).

The role of the sigB operon in salicylic acid-mediated effects in vivo. In rabbits catheterized in the aorta and infected with parental S. aureus strain RN6390 or COL or the RN6390 sigB-complemented mutant, salicylic acid therapy yielded significant reductions in vegetation weight, as well as in vegetation and kidney bacterial densities, as compared with their respective untreated controls. In contrast, in rabbits infected with the $\operatorname{sig} B$ deletion mutants of either RN6390 or COL, salicylic acid treatment did not yield any significant effects on these parameters (Table 5). The salutary effects of salicylic acid therapy in animals infected with parental strain RN6390 were closely paralleled in salicylic acid-treated animals infected with its $r s b U$-repaired derivative SH1000 (Table 5). Taken together, these data further confirmed the integral role of the sigB operon in mediating the antimicrobial effects of salicylic acid in vivo.

\section{Discussion}

Over the past decade, a number of investigations have demonstrated that acetylsalicylic acid and/or its predominant metabolite, salicylic acid, exhibit definable impacts both in vitro and in vivo on microbial virulence phenotypes. The following effects have been noted: reduction of adhesion to relevant biomatrices (29), reduction of capsule production (30), mitigation of biofilm formation (31), and diminution of vegetation growth, intravegetation bacterial proliferation, and hematogenous dissemination in experimental infective endocarditis (32). Salicylic acid also has been shown to upregulate the translation of specific gene loci, including multiple antibiotic-resistance loci (33); to induce cytoplasmic proteins (34); and to increase quinolone resistance (35). We recently studied the potential antimicrobial mechanisms of aspirin in the infective endocarditis model (8) and demonstrated that salicylic acid was the principal mediator of these antimicrobial effects in vivo. The present study was designed to further characterize the in vitro and in vivo impact of salicylic acid, particularly focusing on potential microbial genetic pathways involved in these effects, in the context of experimental infective endocarditis.

We demonstrated that, at levels achievable in human serum (11), salicylic acid mitigated two distinct virulence phenotypes that are of key relevance in the pathogenesis of experimental infective endocarditis: matrix protein binding (i.e., to fibrinogen and fibronectin) and $\alpha$-hemolysin activity. These effects were specifically associated with salicylic acid-mediated reductions in the expression of the respective structural genes (i.e., $f n b A, f n b B$, and $b l a)$. Thus, for the fibronectin-binding phenotype, salicylic acid-mediated reduction in binding can clearly be linked to a negative impact on the $f n b A$ and/or fn $b B$ genes. However, the reduction in overall binding of $S$. aureus to fibrinogen likely relates to a multifactorial impact of salicylic acid. For example, the expression of ClfA (a principal fibrinogen adhesin) can be upregulated by sigB (36). Therefore, salicylic acid could potentially either reduce or increase ClfA expression, depending on the growth phase. Moreover, the fibronectin-binding adhesin FnbA can mediate binding to fibrinogen as well as fibronectin (37). Finally, there are other fibrinogen-binding adhesins (e.g., Coa, ClfB) that could also be influenced by salicylic acid treatment. Therefore, the net mitigating effect of salicylic acid on $S$. aureus binding to fibrinogen is likely to reflect the composite impact on sar $A$, fnb $A$, and/or fnbB expression.

In addition to the suppression of matrix protein binding and cytolytic profiles, enhanced exoenzyme and protein A production was observed in the presence of salicylic acid. These pleiotropic phenotypic findings raised the likelihood that salicylic acid executed its antimicrobial effects through one or more global regulatory networks rather than a decrease in general gene transcription. We focused our investigations on three global regulons, $\operatorname{sar} A$, $a g r$, and $\operatorname{sig} B$, since they have each been shown to impact one or more of the above phenotypes and have been implicated in in vivo virulence, including in infective endocarditis $(19,38)$. 
In $S$. aureus, the global regulatory element sarA upregulates the expression of a number of matrix adhesin genes (e.g., $f n b A$ ) and exotoxin genes (e.g., bla), while downregulating certain enzyme genes (e.g., serine protease [sppa] and lipase [geb]) and protein A production (spa) $(13,14)$. The sarA locus is also involved in maximizing activation of the agr operon, an additional key global regulatory locus of $S$. aureus, which is particularly involved in $\alpha$-hemolysin production (e.g., via bla) $(39,40)$. Transcriptional analysis indicates that the sar $A$ locus is composed of three overlapping transcripts, designated sarA P1, sarA P3, and sarA P2 (41), all encoding the $14.5-\mathrm{kDa}$ SarA protein, the major effector molecule of this locus. Recognizing that differential sarA promoter activation may modulate SarA expression, we explored the effects of salicylic acid on both the individual sarA promoters and the entire sarA operon, and also on the promoters of agr RNAII and RNAIII. Northern analysis, fluorometry, and flow cytometry studies demonstrated a salicylic acid-induced suppression of the sarA P1 promoter and of expression of the intact sarA P2-P3-P1 promoter complex, with a concomitant upregulation of the sarA $\mathrm{P} 3$ promoter. As the sarA P1 promoter is the strongest promoter of the sarA locus, this salicylic acid-mediated diminution in its activation correlates well with mitigation of sarA-dependent phenotypes (e.g., adhesin and $\alpha$-hemolysin functions). Our immunoblot studies disclosed a $10 \%$ salicylic acid-mediated reduction in SarA protein expression, consistent with the key role of the sarA locus in this context. The salicylic acid-mediated decrease in SarA protein levels was smaller than the reduction in sarA P1 promoter expression, by transcriptional fusion analyses. These data are concordant with the relative insensitivity of the immunoblot to detect modest differences in gene expression.

Additionally, our in vivo data underscored the significance of sarA in mediating salicylic acid-induced antivirulence effects. Importantly, we showed a suppression of sarA $\mathrm{P} 1$ promoter activity in animals with infective endocarditis treated with aspirin. Moreover, treatment of animals with salicylic acid reduced several key parameters of disease severity (e.g., vegetation weights, and vegetation and renal bacterial densities) in experimental infective endocarditis caused by the parental strain, but not in that caused by an isogenic sarA knockout construct. The inverse relationship between the sarA P1 and the sarA P3 promoter effects in the presence of salicylic acid (i.e., decreased sarA P1 transcription and increased sarA P3 transcription) fits the current paradigm of sarA regulation. Thus, activation of the otherwise quiescent sarA $\mathrm{P} 3$ promoter upstream may interfere with activation of the proximal but stronger sarA P1 promoter. This mechanism is thought to occur either by promoter occlusion or by the binding of a repressor protein between the sarA P1 and the sarA P3 promoter $(28,41)$. These findings provided important clues that the overall antistaphylococcal effect of salicylic acid is likely executed via additional regulatory elements that upregulate the sarA P3 promoter (e.g., $\operatorname{sig} B)(15,16)$. Although the above data clearly implicate sar $A$ in mediation of the salutary impacts of salicylic acid in vivo, we cannot precisely quantify the potential contributions of a large cadre of responsive downstream structural genes in this context.

The salicylic acid-induced suppression of sarA was accompanied by a reduction in agr RNAII and $a g r$ RNAIII promoter expression. It has previously been shown that SarA has binding specificity for the P2 and P3 interpromoter region of agr, thereby activating RNAII and RNAIII transcription (40). Therefore, downregulation of the agr promoters might either constitute a direct salicylic acid-induced effect on the agr locus, or be indirectly mediated via suppression of the sarA locus. Similarly, salicylic acid-mediated reduction in $\alpha$-hemolysin production might be multimodal, i.e., via suppression of agr-dependent or sarA-dependent pathways, since the bla promoter is activated in response to both signals (42).

It should be noted that $S$. aureus virulence parameters in vitro (e.g., matrix protein adhesion and cytolysins) were not completely suppressed by salicylic acid but were reduced, in a drug concentration-dependent manner, by a maximum of approximately $50 \%$. This finding correlates with the relative extents of sarA P1 and P2-P3-P1 promoter suppression, and concomitant sarA $\mathrm{P} 3$ promoter activation by both Northern and fluorometric analyses. It should also be emphasized that, in vivo, tissue bacterial densities were reduced by approximately $1 \log _{10} \mathrm{CFUs} / \mathrm{g}$ in untreated animals infected with an $S$. aureus sarA mutant; in contrast, salicylic acid treatment in animals infected with its sarAintact parent strain yielded approximately $2 \log _{10} \mathrm{CFUs} / \mathrm{g}$ decrease in tissue bacterial densities. These findings suggest that $s a r A$-independent and/or sarA-coregulated pathways are likely involved in salicylic acid-induced suppression of in vivo and in vitro virulence parameters. This notion is supported by: (a) the presence of at least 16 two-component regulatory systems in S. aureus, one or more of which may be responsive to salicylic acid (43); (b) the burgeoning family of sarA homologs that impact $S$. aureus virulence determinants (43); and (c) the numerous (>100) sarA-regulated downstream genes in S. aureus (44). It is therefore not surprising that regulatory pathways other than sarA and agr would be involved in salicylic acid-induced impacts. Studies of the potential effects of salicylic acid on candidate sarA homologs are in progress in our laboratories. Finally, mechanisms other than global regulatory elements for example, direct effects on downstream structural genes and their encoded gene products - might contribute to salicylic acid impacts on $S$. aureus virulence and cannot be excluded.

Alternative $\sigma$ factors have been shown to contribute importantly to bacterial survival by regulating stressresponse genes in response to environmental stimuli (e.g., $\mathrm{pH}$, heat shock), growth phase-specific signals 
(e.g., the stationary phase of growth), and ATP-deprivation conditions (e.g., anaerobic growth) $(45,46)$. As part of the RNA polymerase holoenzyme, alternative $\sigma$ factors in Gram-positive bacteria function to enable the organism to transcribe genes that are activated in response to stresses in hostile environments (e.g., low $\mathrm{pH}$ of abscesses) (38). In S. aureus, the sigB operon has been shown to participate in the transcriptional regulation of $\operatorname{sar} A(15,16,28)$, principally via $\operatorname{sig} B$-dependent activation of the sarA $\mathrm{P} 3$ promoter. We confirmed the salicylic acid-mediated activation of sarA P 3 by two independent in vitro assays, flow cytometry and Northern analysis. In addition, enhancement of the sigB operon was demonstrated fluorometrically by salicylic acid-induced increases in the expression of the sigB-dependent promoter asp23. Furthermore, the key role of the sig $B$ operon in executing the antivirulence properties of salicylic acid was shown in two distinct genetic background strains (RN6390 and COL). In these strains, deletion of the $\operatorname{sig} B$ operon abrogated the salicylic acid-mediated suppression of in vitro matrix protein binding, $\alpha$-hemolysin production, and in vivo $S$. aureus virulence in infective endocarditis. The salicylic acid-mediated mitigation of these phenotypes was restored following $\operatorname{sig} B$ complementation.

Using current genetic paradigms for $\operatorname{sig} B, a g r$, and sarA regulon interactions and their downstream effects on major structural virulence genes, our data sets would suggest the following pathway for salicylic acid-mediated impacts: Salicylic acid activates the $\operatorname{sig} B$ operon, which then stimulates the sigB-dependent sar $A$ $\mathrm{P} 3$ promoter. In turn, sarA $\mathrm{P} 1$ promoter expression is repressed, leading to downmodulation of sarA and the ensuing agr activation. These events would be expected to repress sarA-upregulatable genes (e.g., fnb $b, f n b B$, bla), derepress sarA-downregulatable exoenzyme (e.g., lipase and protease) and protein A genes (e.g., spa), and repress agr-upregulatable genes (e.g., bla).

The sigB operon of $S$. aureus consists of four genes, $r s b U, r s b V, r s b W$, and sigB (45). Studies in Bacillus subtilis indicate that $r s b U$ is involved predominantly in acid stress, while $r s b V$ may mediate $\operatorname{sig} B$ activation in response to other environmental stressors (e.g., drugs) (47). The ability of salicylic acid to activate sigB and its downstream sigB-dependent promoters, sar $A$ P3 and asp23, in strain RN6390 was somewhat surprising, since this strain carries a natural 11-bp deletion within $r s b U$, leading, in vitro, to a state in which sigB is relatively deficient (but not absent) (45). However, a virtually identical degree of salicylic acid-mediated $\operatorname{sig} B$ activation was found in SH1000, the functional $r s b U$ derivative of RN6390, and in FDA486, a strain with an intact $r s b U$ gene. Moreover, salicylic acid exposure yielded similar mitigating impacts on in vitro phenotypes and in vivo virulence events in strain RN6390 and its $r s b U$-repaired variant SH1000, as well as in strain COL, a genetically distinct strain with an intact $r s b U$ locus. These observations emphasize that the effects of salicylic acid may be mediated via an $r s b U$-independent pathway within the sigB operon, and that such pathways may vary between distinct strains.

With the current widespread problem of multiantibiotic resistance in S. aureus, development of new therapeutic agents that can mitigate the activation of bacterial virulence genes required for initiating and propagating infections is considered a high priority. In this respect, the use of aspirin and salicylic acid appears to reduce staphylococcal virulence, rather than inducing bactericidal activity. Provided that a favorable safety profile is established in therapeutic trials of systemic $S$. aureus infection, aspirin may have the potential to be an effective adjunctive agent in the treatment of serious hospital- or community-acquired S. aureus infections.

\section{Acknowledgments}

This work was supported by NIH grants AI-39108 (to A.S. Bayer), AI-39001 and AI-48031 (to M.R. Yeaman), and AI-37142 (to A.L. Cheung), by Deutsche Forschungsgemeinschaft grant KU1155/1-1 (to L.I. Kupferwasser), and by a research Grant-in-Aid from the Western States Affiliate of the American Heart Association (0150699Y to A.S. Bayer).

1. Brumfitt, W., and Hamilton-Miller, J. 1989. Methicillin-resistant Staphylococcus aureus. N. Engl. J. Med. 320:1188-1196.

2. Hiramatsu, K., et al. 1997. Dissemination in Japanese hospitals of strains of Staphylococcus aureus heterogeneously resistant to vancomycin. Lancet. 350:1670-1673.

3. Balaban, N., et al. 1998. Autoinducer of virulence as a target for vaccine and therapy against Staphylococcus aureus. Science. 280:438-440.

4. Shinefield, H., et al. 2002. Use of a Staphylococcus aureus conjugate vaccine in patients receiving hemodialysis. N. Engl. J. Med. 346:491-496.

5. Mahan, M.J., Slauch, J.M., and Mekalanos, J.J. 1993. Selection of bacterial virulence genes that are specifically induced in host tissues. Science. 259:686-688.

6. Kopp, E., and Ghosh, S. 1994. Inhibition of NF-kappa B by sodium salicylate and aspirin. Science. 265:956-959.

7. Bitko, V., Velazquez, A., Yang, L., Yang, Y.C., and Barik, S. 1997. Transcriptional induction of multiple cytokines by human respiratory syncytial virus requires activation of NF-kappa B and is inhibited by sodium salicylate and aspirin. Virology. 232:369-378.

8. Kupferwasser, L.I., et al. 1999. Acetylsalicylic acid reduces vegetation bacterial density, hematogenous bacterial dissemination, and frequency of embolic events in experimental Staphylococcus aureus endocarditis through antiplatelet and antibacterial effects. Circulation. 99:2791-2797.

9. Cheung, A.L., et al. 1994. Diminished virulence of a sar-/agr-mutant of Staphylococcus aureus in the rabbit model of endocarditis. J. Clin. Invest. 94:1815-1822.

10. Wolz, C., McDevitt, D., Foster, T.J., and Cheung, A.L. 1996. Influence of agr on fibrinogen binding in Staphylococcus aureus Newman. Infect. Immun. 64:3142-3147.

11. Needs, C.J., and Brooks, P.M. 1985. Clinical pharmacokinetics of the salicylates. Clin. Pharmocokinet. 10:164-177.

12. Karlsson, A., Saravia-Otten, P., Tegmark, K., Morfeldt, E., and Arvidson, S. 2001. Decreased amounts of cell wall-associated protein A and fibronectin-binding proteins in Staphylococcus aureus sarA mutants due to up-regulation of extracellular proteases. Infect. Immun. 69:4742-4748.

13. Cheung, A.L., and Projan, S.J. 1994. Cloning and sequencing of sarA of Staphylococcus aureus, a gene required for the expression of agr. J. Bacteriol. 176:4168-4172.

14. Cheung, A.L., Koomey, J.M., Butler, C.A., Projan, S.J., and Fischetti, V.A. 1992. Regulation of exoprotein expression in Staphylococcus aureus by a locus (sar) distinct from agr. Proc. Natl. Acad. Sci. U. S. A. 89:6462-6466.

15. Deora, R., Tseng, T., and Misra, T.K. 1997. Alternative transcription factor $\sigma^{S B}$ of Staphylococcus aureus: characterization and role in transcription of the global regulatory locus sar. J. Bacteriol. 179:6355-6359.

16. Manna, A.C., Bayer, M.G., and Cheung, A.L. 1998. Transcriptional analysis of different promoters in the sar locus in Staphylococcus aureus. J. Bacteriol. 180:3828-3836. 
17. Horsburgh, M.J., et al. 2002. $\sigma^{B}$ modulates virulence determinant expression and stress resistance: characterization of a functional $r s b U$ strain derived from Staphylococcus aureus 8325-4. J. Bacteriol. 184:5457-5467.

18. Bhakdi, S., and Tranum-Jensen, J. 1991. Alpha-toxin of Staphylococcus aureus. Microbiol. Rev. 55:733-751.

19. Bayer, A.S., et al. 1997. Hyperproduction of alpha-toxin by Staphylococcus aureus results in paradoxically reduced virulence in experimental endocarditis: a host defense role for platelet microbicidal proteins. Infect. Immun. 65:4652-4660.

20. Menzies, B.E., and Kernodle, D.S. 1994. Site-directed mutagenesis of the alpha-toxin gene of Staphylococcus aureus: role of histidines in toxin activity in vitro and in a murine model. Infect. Immun. 62:1843-1847.

21. Smeltzer, M.S., Hart, M.E., and Iandolo, J.J. 1993. Phenotypic characterization of $x p r$, a global regulator of extracellular virulence factors in Staphylococcus aureus. Infect. Immun. 61:919-925.

22. Cheung, A.L., Nast, C.C., and Bayer, A.S. 1998. Selective activation of sar promoters with the use of green fluorescent protein transcriptional fusions as the detection system in the rabbit endocarditis model. Infect. Immun. 66:5988-5993.

23. Cormack, B.P., Valdivia, R.H., and Falkow, S. 1996. FACS-optimized mutants of the green fluorescent protein (GFP). Gene. 173:33-38.

24. Gertz, S., et al. 1999. Regulation of sigmaB-dependent transcription of sigB and asp23 in two different Staphylococcus aureus strains. Mol. Gen. Genet. 261:558-566.

25. Xiong, Y.Q., et al. 2002. Activation and transcriptional interaction between agr RNAII and RNAIII in Staphylococcus aureus in vitro and in an experimental endocarditis model. J. Infect. Dis. 186:668-677.

26. Cheung, A.L., Wolz, C., Yeaman, M.R., and Bayer, A.S. 1995. Insertional inactivation of a chromosomal locus that modulates expression of potential virulence determinants in Staphylococcus aureus. J. Bacteriol. 177:3220-3226.

27. Cheung, A.L., Bayer, M.G., and Heinrichs, J.H. 1997. sar genetic determinants necessary for transcription of RNAII and RNAIII in the agr locus of Staphylococcus aureus. J. Bacteriol. 179:3963-3971.

28. Cheung, A.L., Chien, Y.T., and Bayer, A.S. 1999. Hyperproduction of alpha-hemolysin in a $\operatorname{sigB}$ mutant is associated with elevated SarA expression in Staphylococcus aureus. Infect. Immun. 67:1331-1337.

29. Farber, B.F., and Wolff, A.G. 1992. The use of nonsteroidal antiinflammatory drugs to prevent adherence of Staphylococcus epidermidis to medical polymers. J. Infect. Dis. 166:861-865.

30. Domenico, P., Schwartz, S., and Cunha, B.A. 1989. Reduction of capsular polysaccharide production in Klebsiella pneumoniae by sodium salicylate. Infect. Immun. 57:3778-3782.

31. Polonio, R.E., Mermel, L.A., Paquette, G.E., and Sperry, J.F. 2001. Eradication of biofilm-forming Staphylococcus epidermidis (RP62A) by a combination of sodium salicylate and vancomycin. Antimicrob. Agents Chemother. 45:3262-3266.

32. Nicolau, D.P., et al. 1993. Reduction of bacterial titers by low-dose aspirin in experimental aortic valve endocarditis. Infect. Immun. 61:1593-1595.

33. Cohen, S.P., Levy, S.B., Foulds, J., and Rosner, J.L. 1993. Salicylate induction of antibiotic resistance in Escherichia coli: activation of the mar operon and a mar-independent pathway. J. Bacteriol. 175:7856-7862.

34. Sun, Z., Cheng, S.J., Zhang, H., and Zhang, Y. 2001. Salicylate uniquely induces a $27-\mathrm{kDa}$ protein in tubercle bacillus. FEMS Microbiol. Lett. 203:211-216.

35. Gustafson, J.E., et al. 1999. Growth in the presence of salicylate increases fluoroquinolone resistance in Staphylococcus aureus. Antimicrob. Agents Chemother. 43:990-992.

36. Wolz, C., Goerke, C., Landmann, R., Zimmerli, W., and Fluckiger, U.
2002. Transcription of clumping factor $A$ in attached and unattached Staphylococcus aureus in vitro and during device-related infection. Infect. Immun. 70:2758-2762.

37. Wann, E.R., Sivashankarappa, G., and Höök, M. 2000. The fibronectinbinding MSCRAMM FnbpA of Staphylococcus aureus is a bifunctional protein that also binds to fibrinogen. J. Biol. Chem. 275:13863-13871.

38. Gertz, S., et al. 2000. Characterization of the $\sigma^{B}$ regulon in Staphylococcus aureus. J. Bacteriol. 182:6983-6991.

39. Kornblum, J., et al. 1990. Agr: a polycistronic locus regulating exoprotein synthesis in Staphylococcus aureus. In Molecular biology of the Staphylococci. R.P. Novick, editor. VCH Publishers. New York, New York, USA. 373-402.

40. Chien, Y.T., and Cheung, A.L. 1998. Molecular interactions between two global regulators, sar and agr, in Staphylococcus aureus. J. Biol. Chem. 273:2645-2652.

41. Bayer, M.G., Heinrichs, J.H., and Cheung, A.L. 1996. The molecular architecture of the sar locus in Staphylococcus aureus. J. Bacteriol. 178:4563-4570

42. Chien, Y., Manna, A.C., Projan, S.J., and Cheung, A.L. 1999. SarA, a global regulator of virulence determinants in Staphylococcus aureus, binds to a conserved motif essential for sar-dependent gene regulation. J. Biol. Chem. 274:37169-37176.

43. Cheung, A.L., and Zhang, G. 2002. Global regulation of virulence determinants in Staphylococcus aureus by the SarA protein family. Front. Biosci. 7:1825-1842.

44. Dunman, P.M., et al. 2001. Transcription profiling-based identification of Staphylococus aureus genes regulated by the agr and/or sarA loci. J. Bacteriol. 183:7341-7353.

45. Kullik, I., Giachino, P., and Fuchs, T. 1998. Deletion of the alternative sigma factor sigmaB in Staphylococcus aureus reveals its function as a global regulator of virulence genes. J. Bacteriol. 180:4814-4820.

46. Hecker, M., Schumann, W., and Volker, U. 1996. Heat-shock and general stress response in Bacillus subtilis. Mol. Microbiol. 19:417-428.

47. Palma, M., and Cheung, A.L. 2001. $\sigma^{B}$ activity in Staphylococcus aureus is controlled by RsbU and an additional factor(s) during bacterial growth. Infect. Immun. 69:7858-7865.

48. Booth, M.C., et al. 1997. Staphylococcal accessory regulator (sar) in conjunction with agr contributes to Staphylococcus aureus virulence in endophthalmitis. Infect. Immun. 65:1550-1556.

49. Wu, S., de Lencastre, H., and Tomasz, A. 1996. Sigma-B, a putative operon encoding alternate sigma factor of Staphylococcus aureus RNA polymerase: molecular cloning and DNA sequencing. J. Bacteriol. 178:6036-6042.

50. Dhawan, V.K., et al. 1997. Phenotypic resistance to thrombin-induced platelet microbicidal protein in vitro is correlated with enhanced virulence in experimental endocarditis due to Staphylococcus aureus. Infect. Immun. 65:3293-3299.

51. Koo, S.P., Bayer, A.S., Sahl, H.G., Proctor, R.A., and Yeaman, M.R. 1996. Staphylocidal action of thrombin-induced platelet microbicidal protein is not solely dependent on transmembrane potential. Infect. Immun. 64:1070-1074.

52. McDevitt, D., Francois, P., Vandaux, P., and Foster, T.J. 1994. Molecular characterization of the clumping factor (fibrinogen receptor) of Staphylococcus aureus. Mol. Microbiol. 11:237-248.

53. Chambers, H.F. 1987. Coagulase-negative staphylococci resistant to beta-lactam antibiotics in vivo produce penicillin-binding protein $2 \mathrm{a}$. Antimicrob. Agents Chemother. 31:1919-1924.

54. Fowler, V.G., Jr., et al. 2000. In vitro resistance to thrombin-induced platelet microbicidal protein in isolates of Staphylococcus aureus from endocarditis patients correlates with an intravascular device source. J. Infect. Dis. 182:1251-1254. 